\title{
A Study of Material Design Methodology of Environmental Art Design
}

\author{
Hu Juan \\ Wuhan City Polytechnic
}

\author{
Zhu Ya-li* \\ Hubei Institute of Fine Arts
}

\begin{abstract}
There are many related papers in the material design of environmental art design, which emphasizes ecological, stylized, cultural self-confident, regional, economic, matching skills, etc. These papers cover the scientific knowledge of the material itself, the epochal spirit of structure, national cultural psychology, historical context, etc. Although there are many aspects involved, there is still no clear methodology for analysis. From the perspective of the teaching objectives of environmental art design, this paper combines with the theory of knowledge sociology to elaborate the material design methodology.
\end{abstract}

Keywords-Metaphor of material itself; "material, craft, model and structure"; "Knowledge" behind the structure; Skills of material design

\section{INTRODUCTION}

Environmental art design refers to a practical art for the integrated design of the space inside and outside the building through art design. Through a series of plans, enclosing means, and artistic treatment of the space interface to meet people's needs in functional use and visual aesthetic. Its design is an extremely comprehensive system behavior that encompasses several subsystems associated with it. It integrates functions, art and technology, and involves many subject contents in art and science. It has the characteristics of multidisciplinary intertwinement, infiltration and integration.

There are many related papers in the material design of environmental art design, which emphasizes ecological, stylized, cultural self-confident, regional, economic, matching skills, etc.. These cover the scientific knowledge of the material itself, the epochal spirit of the structure, the national cultural psychology, the historical context, etc.. Although there are many aspects involved, there is still no clear methodology for analysis. From the perspective of the teaching objectives of environmental art design, this paper attempts to elaborate the material design methodology, combined with the theory of knowledge sociology.

Epistemology claims to be the foundation of all subjects, but in fact it is determined by the state of science at any given time, and the principles used to criticize knowledge are subject to social and historical conditions. Therefore, the central idea of knowledge sociology is social environmental determinism, that is, there is a dominant methodology in every historical stage. This method is subjective and objective. It is difficult for individuals to break away from the macro logic of this methodology. People can have different views on the same issue, but the methodology is difficult to break away from the main era. That is to say, knowledge sociology believes that thought or "knowledge" is ultimately determined by the thinker and the social environment and conditions in which he lives.

China is gradually entering the era of knowledge economy. Knowledge begins to occupy a central position in society, and frequent exchange of various knowledge is faster and the integration is deeper. Things are universally connected, and each point of knowledge goes back to a final subject consciousness. The current technological changes have triggered a series of changes, bringing global strategic austerity, strengthening of high-tech barriers, scale, and integration. The development of the industrial chain is blocked by strategic austerity and protectionism. Therefore, "knowledge" under the different subject consciousness also strengthens the confrontation, and each enriches, perfects, promotes and consolidates its "knowledge" so as to show its powerful vitality in the next cycle.

In this context, environmental art design in China should also adjust its "knowledge" to cope with future development. The characteristics in the material design methodology are as follows. The first one is the "knowledge" of the structure has a clear subject consciousness. The second one is future-oriented material design. The third one is the multidisciplinary intertwinement, infiltration and integration of material design.

\section{DETERMINANTS OF MATERIAL DESIGN}

\section{A. "Knowledge" behind the structure}

T. S. Eliot said: "The only way to express emotion through art form is to find an objective correlative." The objective correlative refers to a series of objects that can trigger a certain emotion and direct sensory experience. Reflected in environmental art design, it is the formation of "knowledge" through the structure constructed by materials. For example, in our traditional structure aesthetic, material design pursues "thin, wrinkled, leaky, and translucent". It is hoped that the structure created by this material design points to a "knowledge" characterized by self-discipline, asceticism, meticulousness, richness, introversion, etc.. This "self-care" posture of the structure, even if it is not clear what it represents, may know that it has something with religion. A spiritual pursuit, more of a confrontational posture, is agitating, and infecting viewers. So the expressions of different structures point to different "knowledge" and express a clear subject consciousness. 
How to understand and define the "knowledge" behind the structure is key to successful design. In general, the appearance of structures can be divided into three categories: thin, fat, and light mass with expansion nature. For example, the traditional structures in China are generally thinner, with a clear skeleton, a tall and straight shape, a heavy mass, etc.. The traditional structures in Europe, the United States and the Mediterranean region are generally fatter, with strong envelopment form, giving people a sense of warmth, embrace, acceptance and indulgence. The traditional Japanese structures, with the combination of the East and the West, has formed its own characteristics of light mass with expansion nature. Compared with traditional Chinese structures, Japanese structure is generally lighter, especially the part of foot. Compared with the West, Japanese is more divergent and expands outward. These structure features are components of the "knowledge" of different regions and represent their own clear subject consciousness. People build, enrich, and develop their own "knowledge" under different subject consciousness which also has its own objective correlative. To evaluate the quality of these objective correlatives also rests with whether they can reflect the subject consciousness better or not. As to environmental art design, the better method of expressing the subject consciousness is the most appropriate material design.

\section{1) The most appropriate material design}

The works of Zaha Hadid, a famous architect, tend to be futuristic designs which are full of the regional characteristics. She always reminds people how the local wilderness itself crosses the hills, how the caves carry out, how the river is zigzag, and how the mountain leads the direction. Her works are always closely combined with the region, the landscape, and the humanities to achieve its appropriate posture. There are many different main materials in her works, such as concrete, GRG (Glassfiber Reinforced Gypsum), titanium-zinc plate, etc. But no matter how materials change, the futuristic, fluidity, and guidance of its structures have not changed. A mature designer will show his own stable "knowledge" regardless of the project's intention, material technology limitations, and geographical differences. The most appropriate material design is not a fixed system, but the most suitable "knowledge" choice based on different situations, the development of the era technology, and popularity, etc..

\section{2) Metaphor of material itself}

Designers have their own preferred materials when designing because materials themselves are metaphorical. For example, titanium-zinc plates, with clear metal wire drawing, are mainly calm blue-gray, supplemented by several hard and cold metal colors. The structures constructed with them may have a strong and deep metallic appearance. $80 \%$ of the roofs in Paris are made of this material, which has added the profundity to the city. Brass is characterized by a kind of "nostalgic comfort", and for a long time the patina has appeared which can show a beautiful vicissitudes of life. Many artists and designers have discovered that glass has a philosophical Zen. It exists, while it does not exist. It is strong and meanwhile it is fragile. It can be seen that the metaphor of material itself becomes a very important choice for designers to express their own "knowledge".

\section{3) Ultimate goal of material design}

In environmental art design, as an objective correlative of subject consciousness, structures achieve the ultimate goal through materials. Therefore, the principle of material design is to express the subject consciousness profoundly, correctly and completely; to conform to the overall mien of the structure; and to guide viewers to understand the "knowledge" of the structure.

\section{B. Future-oriented material design}

Material designers should consider the social responsibility of "knowledge" and the frontier of industry development, therefore, they should also consider the following principles:

(1) Low carbon emissions of materials in total: the total carbon emissions of materials are low in the production process, loading, unloading, transportation, construction and recycling. In the case of low total carbon emissions, it is also possible to further develop its technical natures, such as further development of materials to be biological, integrated and intelligent in the process. It is easy to disassemble and assemble in transport. Use fabricated materials in construction.

(2)Focus on physicochemical characteristics of materials: high strength, good durability, thermal insulation, sound insulation, good energy-saving, wide environmental adaptability, composition, etc., so that structures can reduce its own energy consumption.

With the adjustment of technology, future demand, resources structure and others, the application of composite materials will be more extensive.

\section{Multidisciplinary intertwinement, infiltration and integration of material design}

Material design can be carried out in many aspects, such as process, loading, unloading, transportation, construction, recycling, etc. For example, perforated leather with high differentiation combines strength and hardness, and it can stand up without any specific structural support. Loofah sponge is an ideal acoustic insulator. It can reabsorb and recycle toxic industrial wastewater from denim dyeing to become a beautiful and harmless interior wall decoration. It is also a good acoustic material. Therefore, the future of material design will be intertwined, infiltrated, integrated with diverse fields and professions.

\section{PRINCIPLES OF MATERIAL DESIGN}

After clarifying the determinants in material design, the next is to analyze the basic principles of material design. There are many materials involved in environmental art design, which can be classified into the following:

(1) Structural materials include wood, bamboo, stone, cement, concrete, metal, brick, ceramic, glass, engineering plastics, composite materials, etc. (2) Decorative materials include coatings, paints, veneers, tiles, glass with special effects, etc. (3)Special materials are used for waterproofing, moisture proof, anticorrosion, fireproofing, flame retardant, sound insulation, heat insulation, heat preservation, sealing, etc. The materials available are complex and varied, and structural 
materials are often used directly as decorative materials. How to apply them effectively?

The core is to follow the principle of mutual restraint and mutual promotion among the four elements: material, craft, model and structure. Firstly, the choice of materials must meet the structural requirements. If the material is tensile but not compression resistant, it cannot be used as load-bearing material. The relationship between materials and structure is the core of material design. CFRP (Carbon Fiber Reinforced Polymer) bring the light mass and high strength to the extreme. The strength of CFRP is 20 to 50 times that of steel. Therefore, the use of CFRP will greatly reduce its weight of the structure and increase its span. For example, the huge "HOME" button on the top of the structure of Apple headquarters in Cupertino, Calif., consists of 44 panels measuring approximately $21.3 \mathrm{~m}$ long and $3.35 \mathrm{~m}$ wide, weighing a total of 80 tons. The roof with that weight can be hoisted completely in construction. If they were replaced by steel plates, the roof, with the weight of 320 to 480 tons, would seem to be too fat. Precisely because CFRP is light mass and high strength, it is possible to have this "floating" roof which can be propped up by the surrounding glass structure. Furthermore it is better to express the "knowledge" behind the structure appropriately.

Secondly, material craft must conform to the characteristics of material itself. For example, bamboo is easy to split, so it is better not to drill the bamboo. And stone is fragile, so sharp corners of stones must be avoided. Because of the metaphor of material itself, the choice and matching of materials will largely determine the mien of the structure. In the same way, craft, model and structure are also restricted by the other three. When a breakthrough is made in one of the elements, it will also drive the leap of the other three elements.

\section{SKILLS OF MATERIAL DESIGN}

With so many materials, is there a rapid selection after clear determinants and design principles? Based on the above exposition, skills of material design can be divided into the following aspects. In general it can be summarized as to be static is to be dynamic which is the core. That is to say, although the space is static and still, a dynamic movement can be achieved with the help of various materials through light and shadow, emptiness and fullness, concave-convex, etc.

(1) It conflicts with the metaphor of material itself. It seems to be solidness in softness and softness in solidness. For example, concrete has the characteristics of cold and alienation, but Tadao Ando makes concrete warm to be like the wood. (2) The transition of materials is the key to the smoothness of the design. The connection between different materials, the transformation and junction between similar materials also define the shape. (3)Transparency of the material is preferred in the small space, however non-transparency is better in the large space which can be divided into several parts or blocks. (4)Use physical and chemical means and others to make materials have its unique metaphor. With the changes of resources, labor and environment, composite materials will be more inclined to be used in the future. However, people prefer natural materials because of its unique characteristics. Therefore, the development of composite materials which are combined with the characteristics of natural materials will have broad prospects. (5)Use the splicing, stacking, etc. to maximize the characteristics of materials. The splicing of different materials, such as wood and resin, can make the structure seem to be stone-like. The overlapping of different modular materials can bring vivid visual effects, such as bricks combined with glass bricks, etc. (6)The relationship between materials and structure is more diversified. Different parts of traditional structures. e.g. roofs, supports, doors and windows, are built of different materials. However, the material application of many excellent cases does not distinguish these components, which are integrated. The structures are wrapped with the same material, which makes them look more futuristic and industrial. (7) Strengthen cross-professional cooperation to develop more materials which reflect unique cultures. Material development is intertwined, infiltrated, integrated with diverse fields and professions, such as integration of ground heating floor, intelligent countertop materials in kitchens, self-heal biological nature of concrete materials. These skills all reflect the vast space of material design.

\section{CONCLUSION}

The development of material design correspondingly puts higher requirements on design, and brings new leap and new forms to design. The "knowledge" with continuous development and improvement can be achieved based on relevant materials and crafts. They mutually promote the development of materials science, the advancement and innovation of craft technology. In the undergraduate teaching of environmental art design, we should pay attention to the construction of related courses of materials design, strengthen multi-disciplinary exchanges and integration, and lay the foundation for the future development.

\section{ACKNOWLEDGMENT}

This paper is the research result of "Teaching Research on Materials Artistic Application of Environmental Art Design" (No. 201613) of Hubei Institute of Fine Arts.

\section{REFERENCES}

[1] Sun Dong-sun. Thought Speech and Culture [J]. Contemporary Rhetoric, 2013(5).

[2] Wang Hu-ning. Political life [M]. Shanghai: Shanghai People's Publishing House, 1995.

[3] Liu Guan-zhong. Design Methodology[M]. Beijing: Higher Education Press, 2011.

[4] Osvald Siren. Chinese Sculpture from the Fifth to the Fourteenth Century [M]. New York: Charles Scribnerl's Sons, 1925. 